\title{
Neurotransmitter excitatory amino acids in synovial fluids demonstrate distinct temporal fluctuations in active arthritis
}

\author{
Terry A McNearney ${ }^{1}$ Niti Goel $^{2}$, Jeffrey R Lisse ${ }^{3}$ and Karin N Westlund ${ }^{4 *}$ \\ ${ }^{1}$ Department of Internal Medicine, University of Texas Medical Branch, Galveston, TX \\ ${ }^{2}$ Kesar Life Sciences, San Francisco, CA \\ ${ }^{3}$ Eli Lilly and Co., Indianapolis, IN \\ ${ }^{4}$ Department of Anesthesiology \& Critical Care Medicine, University of New Mexico School of Medicine, Albuquerque, NM
}

\begin{abstract}
Objective: This case study presents concentrations of synovial fluid (SF) neurotransmitter excitatory amino acids (EAA) glutamate (Glu) and aspartate (Asp) and tumor necrosis factor-alpha (TNF $\alpha)$ in patients with acute onset and chronic arthropathies over time.

Methods: SF samples were sequentially obtained daily from both knees of a patient with acute clinically distinct flares of pseudogout. Two SF samples (same knee) were also obtained from 3 rheumatoid arthritis (RA) patients during active flares. SF EAA and other amino acids (AA) were measured by HPLC, TNF $\alpha$ levels by immunoassay, and SF WBC counts by hemocytometer. SF EAA, other AA and TNF $\alpha$ levels are presented as ratios derived from matched cadaveric SF samples with no antemortem history of active arthritis.
\end{abstract}

Results: Sequential SF Glu and Asp levels from 3 of 4 patients demonstrated impressive fluctuations over time spans from days to years. SF Glu, Asp and serine (Ser) levels were several-fold to higher than other SF AA levels from the patient with pseudogout flares and demonstrated fluctuations over days. Increases in SF WBC counts and Glu levels preceded increases in SF TNF $\alpha$ levels and WBC counts also showed temporal fluctuations.

Conclusion: SF neurotransmitter Glu and Asp level fluctuations were independent of other AA levels or other joint compartments. SF EAA Glu and Asp may reflect and/or further impact ongoing inflammatory intra-articular processes. Further studies will elucidate their utility in arthritis as biomarkers or as novel antiinflammatory targets. An understanding of their fluctuations in acute arthritis could inform the management of joints in the immediate post-trauma setting.

\section{Introduction}

Neurotransmitter excitatory amino acids (EAA) glutamate (Glu) and aspartate (Asp) may play a role as initiators or potentiators of the adaptive immune responses leading to inflammation in experimental and human synovitis [1-3]. In a previous study using single time point collections of repository fluids, we found mean synovial fluid (SF) neurotransmitter Glu and Asp levels corrected to age matched nonarthritic cadaveric SF samples (Patient: Cadaver concentration ratios) were 4.61-fold and 2.15-fold above other nonspecific amino acids (AA), respectively, for all reported arthritis categories [4]. Mean SF Glu and Asp concentrations were highest in the robustly inflammatory arthropathies, e.g., Reiter's syndrome, systemic lupus erythematosus, septic arthritis, rheumatoid arthritis (RA) and acute crystal arthropathies (gout). Conversely, the lowest mean SF Glu and Asp concentrations were obtained from arthropathies with generally lower inflammatory indices, e.g., osteoarthritis (OA) and mild trauma cases.

We hypothesized that SF EAA and tumor necrosis factor alpha (TNFa) levels in flares of active human synovitis would fluctuate, reflecting dynamic microenvironments of the targeted joint space, as noted in inflammatory arthritis models $[2,3,5,6]$. In the present study, a hospitalized patient was convalescing from an acute medical illness when she abruptly developed acute, de novo, bilateral, knee synovitis. These arthritic pseudogout flares diagnosed for each knee were clinically distinct from the other knee for which she underwent daily, sequential, bilateral knee arthrocenteses. The arthritic flares resolved within two weeks of presentation. Additionally, two SF samples from 3 patients during active RA flares, collected at time point ranges spanning months to years were assessed to represent concentration fluctuations over time in the setting of a human chronic arthropathy.

\section{Materials and Methods}

\section{Patients}

Synovial fluid samples were collected and stored from patients who underwent diagnostic or therapeutic arthrocenteses as part of the Gulf Coast Arthritis Registry-Serology, (GCARS) at the University of

${ }^{\star}$ Correspondence to: Karin Westlund High, Department of Anesthesiology \& Critical Care Medicine, University of New Mexico School of Medicine, Albuquerque, NM, Tel: 5052727417; E-mail: khigh@salud.unm.edu

Key words: glutamate, aspartate, serine, TNFa. Neuroinflammation

Received: November 28, 2018; Accepted: December 05, 2018; Published: December 11, 2018 
Texas Medical Branch, Galveston, TX. The samples and medical record reviews were obtained under patient consent and IRB approval. The SF samples were obtained at diagnostic or therapeutic arthrocenteses of the knee from the case study and three patients with rheumatoid arthritis (RA), diagnosed by ACR criteria [7]. Tissue processing for synovial fluids has been previously described [4]. Briefly, synovial fluids were collected, aliquoted in preservative-free tubes and stored at $-80^{\circ} \mathrm{C}$ after cell pelleting.

\section{Acute pseudogout samples}

A 71-year-old female was admitted to the hospital for intravenous (IV) antibiotic treatment of pneumonia. On hospital day 1, blood cultures grew out Streptococcus viridans and a coagulase negative Staphylococcus species. The patient's symptoms of pneumonia improved and on hospital day 7, all antibiotics were discontinued. On day 8, the patient developed acute arthritis of the left knee, with increased warmth, swelling, erythema and severe pain. Left knee arthrocentesis yielded SF WBC count of $54,000 / \mathrm{mm}^{3}$ with $88 \%$ polymorphonuclear leukocytes. Intravenous antibiotics were re-instituted, and the patient was therapeutically arthrocentesed daily over an additional three days (Days 9, 10 and 11), to cover the possibility of a septic knee. Synovial fluid analyses yielded negative bacterial culture results for all samples but intracellular calcium pyrophosphate dihydrate (CPPD) crystals were detected by polarizing microscope examination on Days 9 and 11. The left knee arthritis demonstrated an improved clinical response to reinstitution of IV antibiotics and daily therapeutic arthrocentesis.

Evidence of swelling of the patient's right knee was clinically evident on hospital day 10. Compared to the left knee, the right knee had minimal warmth, less swelling, no erythema and was mildly painful. Blood and right knee SF cultures obtained were negative for bacterial organisms. On Day 10, the right knee SF had a WBC count of $4,500 / \mathrm{mm}^{3}$ and intracellular CPPD crystals were detected on polarizing microscopic examination. On Days 10 and 11, the right knee was treated with daily arthrocentesis and continued IV antibiotic treatment. The patient reported worsening pain levels in the right knee and showed no clinical improvement. On Day 12, the patient was treated for acute pseudogout with low dose oral prednisone and had prompt resolution of the right knee arthritis. The Rheumatology inpatient consult service reported the following clinical diagnoses: left knee, acute pseudogout and the right knee, acute pseudogout, although the patient was initially covered for the possibility of septic arthritis. At one year follow up there had been no recurrence of arthritis in either knee. The clinical diagnosis and treatment are in agreement with ACR and EULAR recommendations [7-9] and a similar clinical presentation has been reported for acute pseudogout [10].

\section{Chronic rheumatoid arthritis samples}

Synovial fluid samples from 3 female patients with RA were collected during active flares. The onset of RA occurred between 1-5 years prior to the first SF sample collection and represents a chronic inflammatory arthritis. Two time points were collected, spanning 0.2 years in RA Patient (Pt) 1; 1.5 years in RA Pt 2 and 9 years in RA Pt 3.

\section{Acute reiter's syndrome samples}

A patient with HIV-1 infection admitted to the hospital for evaluation and treatment of acute onset of fever, severe joint pain and polyarthritis had 4 days of bilateral arthrocenteses. The patient had a similar prior history of fever, severe joint pain and polyarthritis. He was diagnosed and treated for reactive arthritis, or acute Reiter's syndrome.

\section{Cadaveric control samples}

Synovial fluid was harvested from cadavers to serve as nonarthritic controls (4). SF was obtained within 24 hours of witnessed death from patients with no antemortem history of arthritis from clinical examination or medical chart review. Ten cadaveric samples were selected as age-matched controls for SF amino acid levels. Four samples were selected for the control average SF TNFa level. SF WBC counts obtained at arthrocentesis from the nonarthritic cadaveric controls were negligible.

\section{High pressure liquid chromatography (HPLC)}

Free amino acid (AA) determinations were obtained with a Waters 717 HPLC system with autosampler (Waters, Milford, MA, USA), Beckman 114M solvent delivery pumps (Beckman Fullerton, CA, USA), Bioanalytical Systems FL-45 fluorescence detector (excitation $250 \mathrm{nM}$, emission 456: Bioanalytical Systems, West Lafayette, LA, USA) and Waters Millennium software. A standard HPLC protocol was utilized (4). Samples $(100 \mu \mathrm{l})$ were injected into the HPLC analyzer with the reagent solution (sodium borate, $0.01 \mathrm{M}, \mathrm{pH} 8.95$, Millipore filtered) and run at $25^{\circ} \mathrm{C}$.

\section{Experimental standards and quality control}

A detailed description of standards and quality controls have been previously published [4]. The internal AA control was norleucine. The SF samples were thawed once at the time of the assay. The 6 free AA assayed and presented are neurotransmitter EAA glutamate (Glu) and aspartate (Asp), inhibitory amino acids (IAA): serine (Ser) and glycine (Gly) and AA to serve as metabolic controls: arginine (Arg) and threonine (Thr).

The concentrations $(\mu \mathrm{M})$ of AA measured by HPLC were run in triplicate and showed less than $10 \%$ variance of free amino acid concentrations. SF AA in this study are expressed as Patient: Cadaver concentration ratios to correct to concentrations found in normal, nonarthritic joints [4].

\section{TNFa quantification}

The SF TNFa levels were obtained using an immunoassay kit (Quantikine, R\&D Systems, Minneapolis, MN). Average levels from duplicate samples were expressed as the mean SF TNFa Patient: Cadaver concentration ratios. These were derived from the patient SF TNF $\alpha$ concentrations $(\mathrm{pg} / \mathrm{ml}$ ) divided by the mean SF TNF $\alpha$ levels of 4 normal, non-arthritic cadavers. The mean SF TNFa concentration from the cadavers was $1.25 \mathrm{pg} / \mathrm{ml}$, (range: $0-5 \mathrm{pg} / \mathrm{ml}$ ).

\section{Cell counts}

WBC counts derived from patient SF at the time of arthrocentesis were determined by the hospital laboratory in the course of the patient's clinical care and were part of the medical record. WBC counts are expressed as the cell number $/ \mathrm{mm}^{3}$.

\section{Data expression and statistical analyses}

As noted above, mean SF AA and TNFa values are expressed as mean SF Patient: Cadaver concentration ratios. Analyses and presentation of SF mediator fluctuations were performed using Statistica (Tulsa, OK) and SigmaPlot (SPSS, Inc, Chicago, IL) software.

\section{Results}

Figures $1 \mathrm{~A}$ and $1 \mathrm{~B}$ demonstrate mean SF EAA (Glu and Asp) Patient: Cadaver concentration ratios for serial arthocenteses derived 
from both knees of the acute pseudogout patient, who had no previous history of arthritis. During the active flares, SF EAA Glu and Asp concentration ratios and fluctuations were usually 2-3-fold greater than the fluctuations of Arg and Thr, which served as metabolic AA controls. The mean SF EAA concentration ratios were higher in the more clinically symptomatic left knee, on the two days where bilateral arthrocenteses were performed. There were increasing and decreasing fluctuations in mean SF EAA concentration ratios over the 3 or 4 days of daily arthrocentesis: left knee, Days 9 vs 11, (Glu: 36.7 vs 18.6 and Asp: 167 vs 17.7, respectively) and right knee, Days 10 vs 12 (Glu: 3.34 vs 12.35 and Asp: 4.8 vs 17.3, respectively, Figures 1A and 1B). Also, there were several-fold differences in mean SF EAA concentration ratios between left and right knees from simultaneously drawn same day samples (Days 10 and 11). Mean SF Glu concentration ratios from left and right knees fluctuated 7.8- to 1.8- fold on Days 10 and 11, respectively (Fig. 1A). Mean SF Asp concentration ratios between left and right knees fluctuated 12.29- to 1.71-fold on Days 10 and 11, respectively (Figure 1B).

In the patient with acute pseudogout, mean Patient: Cadaver concentration ratios for SF Ser (Figure 1C) had similar sequential fluctuations as those seen for SF Glu (Figure 1A). There were 2-4-fold differences over 4 days in mean SF Ser concentration ratios in the same knee, left knee, Day 9 vs 11, (44.26 vs 14.4, respectively) and right knee, Day 10 vs 12 (4.65 vs 17.3, respectively). Mean SF Ser concentration ratios from simultaneously drawn same day samples fluctuated from 5.55- to 1.15-fold on Days 10 and 11, respectively (Figure 1C).

The mean Gly, Arg and Thr Patient: Cadaver concentration ratios demonstrated more modest fluctuations in both knees, (Figures 1D1F). There were 1.5-3.6-fold differences over 4 days in mean SF Gly concentration ratios in the same knee, left knee, days 9 vs $11,(12.3$ vs 7.31, respectively) and right knee, Days 10 vs 12 (4.5 vs 13.3), respectively, Figure 1D). The mean Patient: Cadaver concentration ratios for SF Arg ranged between 0.03-13.01 in the left knee and 2.515.74 in the right knee (Figure 1E). The mean SF Thr Patient: Cadaver concentration ratios ranged between 6.14-12.6 in the left knee and 2.445.76 in the right knee (Figure $1 \mathrm{~F}$ ).

Mean SF TNFa Patient: Cadaver concentration ratios were also obtained. The mean SF TNFa Patient: Cadaver concentration ratios were 1.8-3.7-fold higher in the left knee compared to the right (29.470 vs 12.1-13.6, respectively Figure $1 \mathrm{G}$ ). The highest mean SF TNFa concentration ratio was noted on Day 11 in the left knee, two days after the peak SF EAA concentration ratios seen on Day 9. In the right knee, the lower mean SF TNFa Patient: Cadaver concentration ratios did not demonstrate a fluctuating pattern over the three days of serial arthrocentesis.

The left knee SF WBC counts were $54,000 / \mathrm{mm}^{3}, 30,600 / \mathrm{mm}^{3}$, $9,000 / \mathrm{mm}^{3}$ and $5,000 / \mathrm{mm}^{3}$ on Days $8,9,10$ and 11 , respectively. The lowest SF WBC value in the left knee was seen on Day 11, (5000/ $\mathrm{mm}^{3}$ ), four days after parenteral antibiotics were reinstituted and after serial therapeutic arthocenteses. The SF WBC counts from right knee SF samples increased over Days 10,11 and $12\left(4,500 / \mathrm{mm}^{3}\right.$, $19,000 / \mathrm{mm}^{3}$ and $23,000 / \mathrm{mm}^{3}$, respectively, Figure $1 \mathrm{H}$ ). After low dose corticosteroids were administered on Day 12, the right knee arthritis resolved. In the right knee, similar fluctuations and increasing levels were seen in SF Glu, Asp, Ser levels and WBC counts over Days 10-12. The fluctuations in SF WBC counts were not uniformly consistent with the SF EAA concentration ratio fluctuations (Figs. 1A and 1B) or mean SF TNFa concentration ratio fluctuations (Figure $1 G$ ).
Figure 2 illustrates the mean SF EAA (Figures 2A and 2B) IAA and other SF AA (Figures 2C-2F) Patient: Cadaver concentration ratios from three patients with RA at two time points during flares of clinically active arthritis. There were temporal fluctuations in mean SF Patient: Cadaver concentration ratios of 1.5-2-fold for Glu and 1.2-5.5-fold for Asp (Figures 2A and 2B). The mean SF Ser, Gly and Thr Patient: Cadaver concentration ratios were much lower and more constant compared to the mean SF Glu and Asp concentration ratios in the patients with RA (Figures 2C and 2D). Mean SF Arg Patient: Cadaver concentration ratios fluctuated 4-fold in RA Pt 3, although SF concentration ratios of RA Pts 1 and 2, with less time between SF sample collection, were lower and remained more constant between the two arthrocentesis (Figure 2E). Mean TNFa Patient: Cadaver concentration ratios fluctuated severalfold between time points and the RA patients (Figure 2G).

The SF WBC counts from five time points in the $3 \mathrm{RA}$ patients ranged from $13,000-29,375$ cells $/ \mathrm{cm}^{3}$, consistent with inflammatory synovial effusions expected from patients with active RA (Figure $2 \mathrm{H}$ ). The fluctuations in SF WBC counts were not uniformly consistent with the SF EAA concentration ratio fluctuations in RA Pts 1 and 3, (Figures $2 \mathrm{~A}, 2 \mathrm{~B}$ and $2 \mathrm{H}$ ) or mean SF TNFa concentration ratio fluctuations (Figure 2G).

In comparing Figures 1 and 2, there were noted differences in SF EAA and Ser concentration ratios between the RA patients and the patient with acute pseudogout flares. The highest SF Glu concentration
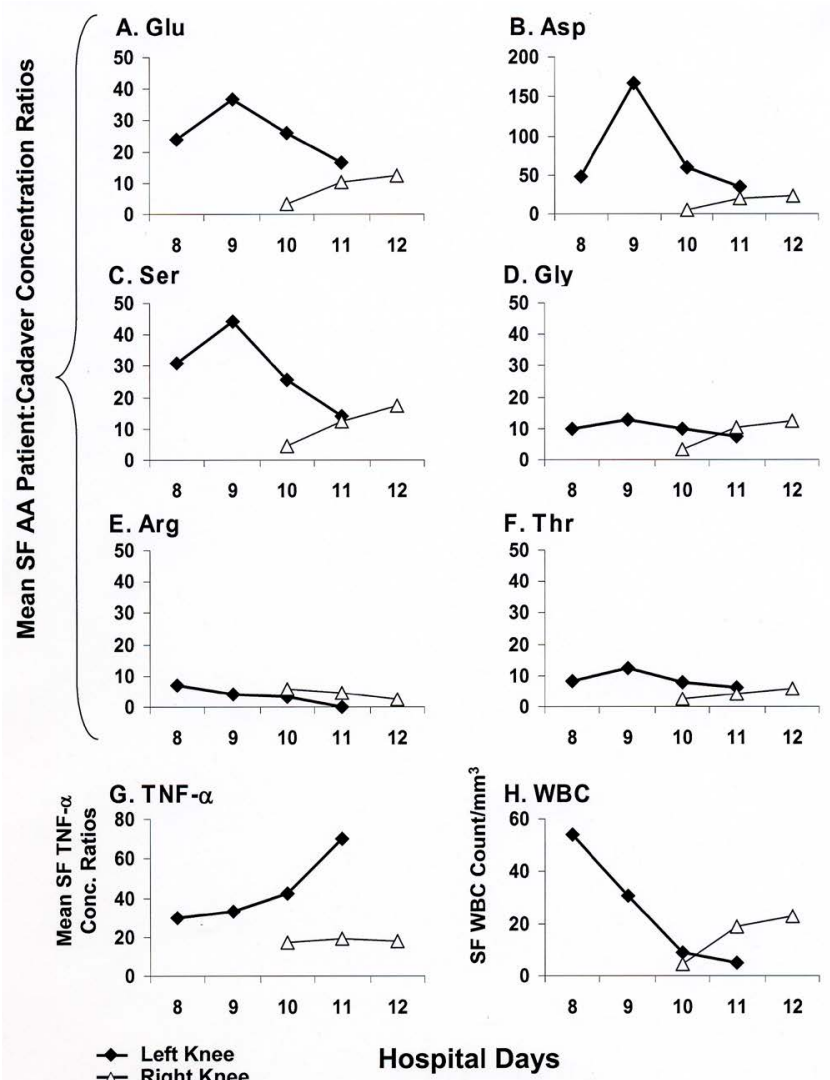

Figure 1. SF EAA and TNF $\alpha$ demonstrate distinct and staggered temporal fluctuations in acute arthritis. Mean SF EAA, other amino acids (AA's), TNF $\alpha$ Patient: Cadaver concentration ratios and white blood cell (WBC) counts were obtained sequentially from a patient with acute pseudogout over 3-4 days from both knees. A: glutamate (Glu); B. aspartate (Asp); C: serine (Ser); D: glycine (Gly); E. Arginine (Arg); F. Threonine (Thr). Mean SF TNF $\alpha$ Patient: Cadaver concentration ratios (G) and SF WBC counts $\left(\times 10^{3} / \mathrm{cm}^{3}\right.$, H) were also obtained 

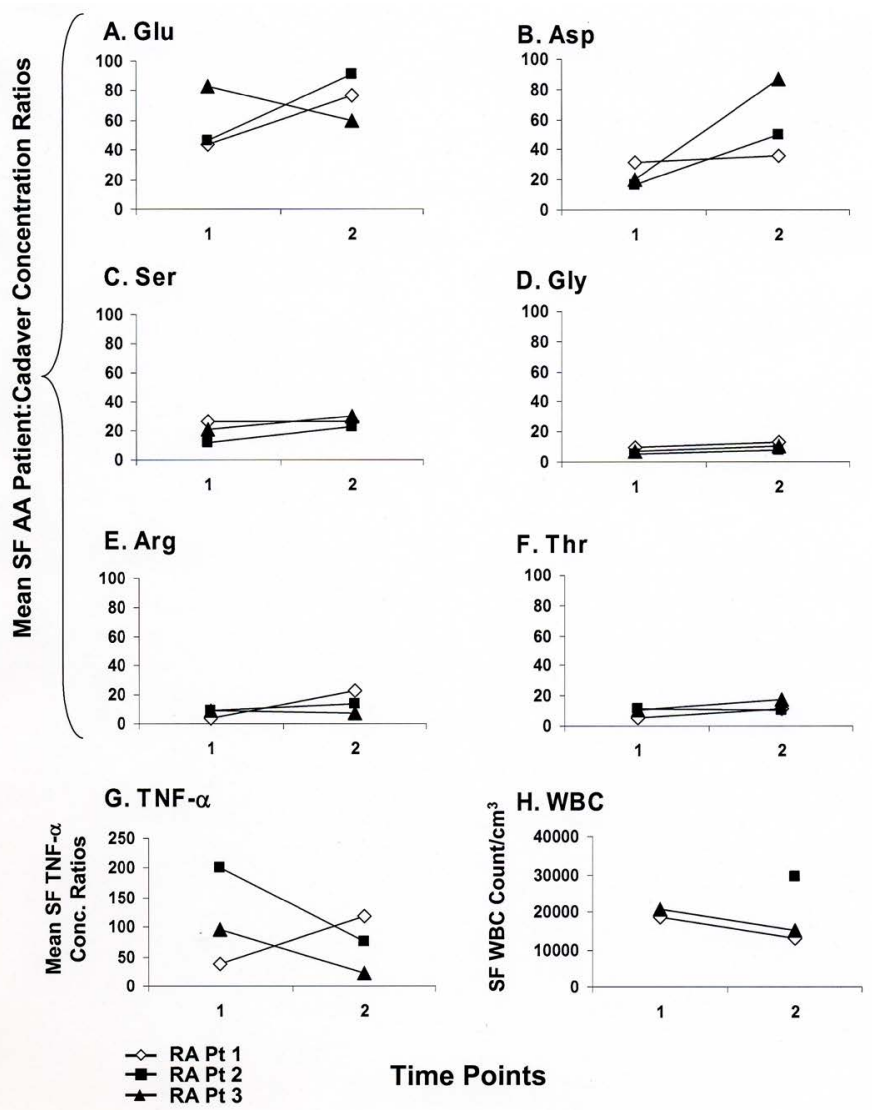

Figure 2. Mean SF EAA, IAA, other AA and TNF $\alpha$ Patient: Cadaver concentration ratios of 3 RA patients at two independent time points during symptomatic flares were obtained. A-H: Noted as in Figure 1. A: glutamate (Glu); B. aspartate (Asp); C: serine (Ser); D: glycine (Gly); E. Arginine (Arg); F. Threonine (Thr). The data are presented as mean SF ratios for the ipsilateral knee of each patient over two arthrocenteses, with differing time spans: Pt 1: 0.2 yrs, Pt 2: 1.5 yrs and Pt 3: 9 years

ratios were 2-fold higher in the RA patients compared to the highest value noted in the pseudogout patient (left knee, Day 9). The highest SF Asp concentration ratio was twice as high in the pseudogout patient (left knee, Day 9) as the highest ratios noted in the RA patients. Similarly, the highest SF Ser concentration ratio in the pseudogout patient was 2 -fold greater than the highest Ser ratio values noted in the RA samples. The ranges of mean SF TNFa Patient: Cadaver concentration ratios, (range: 16.4-250, Figure 2G) were higher in the RA patients, compared to those samples derived from the patient with acute pseudogout (range 29.4-70, Figure 1G). The mean SF TNFa concentration ratio fluctuations between the two time points in the RA patients were not uniformly consistent with the SF EAA concentration ratio fluctuations or SF WBC counts.

The patient with HIV-1 infection had continued fever, polyarthritis and severe joint pain. Arthrocenteses were performed on both knees and left ankle. The patient was diagnosed and treated for reactive arthritis, or acute Reiter's syndrome and had an earlier episode of fever and polyarthritis. Assessment for SF EAA Patient: Cadaver concentration ratios indicated more consistent ratios, within 2-fold among knees and ankle joints over a 4 day period, (SF Glu ratio range $=20-25$; SF Asp ratio range=13-18).

\section{Discussion}

This descriptive study was the result of the opportunity to obtain daily serial SF samples in a patient with acute pseudogout, having a very unusual presentation of acute de novo onsets and two distinctly evolving arthritic courses. SF samples were also collected over different time spans and diagnoses from patients with chronic RA and acute Reiter's arthritis to compare fluctuation patterns of SF EAA and TNFa levels in other acute and chronic inflammatory arthritis. The acute pseudogout and RA patients demonstrated independent, temporally and clinically distinct arthritis courses which were reflected in the different fluctuation level patterns of SF EAA.

The highest SF Glu and Asp concentration ratios in the patients with chronic RA were greater than those noted in the acute pseudogout patient. The other SF amino acid levels were sustained at comparatively much more modest and consistent elevations, possibly reflecting compensatory mechanisms, or equilibration to a new metabolic baseline in the chronic setting. These data are in agreement with a previous report demonstrating higher mean SF Patient: Cadaver concentration ratios from RA Patients than those of other SF AA or compared to SF EAA values from patients with acute pseudogout [4]. The mean SF TNFa concentration ratios obtained over time from the RA patients were generally several-fold higher than those of the acute pseudogout patient. Underlying, compensatory or progressive mechanisms of injury, structural damage, comorbid conditions, or medications may be contributing to the cytokine levels in chronic inflammatory arthropathies. The disease course and SF EAA concentration ratios from the patient with acute Reiter's polyarthritis further supported the observation that SF EAA concentration ratios and clinical courses reflect the microenvironment and local processes of the involved joint, which may be dependent or independent of systemic influences. Fluctuating EAA levels between body compartments of plasma and synovial fluid in the same patient in a variety of arthropathies has been previously reported [11]. Patients with Reiter's or psoriatic arthritis flares have demonstrated the highest SF Glu and Asp concentration ratios [4], a finding which is generally consistent in this report. In a previous crosssectional study, elevated SF Glu and Asp levels in patients with RA, acute gout and OA correlated with elevated SF levels of RANTES, MIP$1 \alpha$, and IL-8. SF EAA levels did not directly correlate with SF TNFa levels [12], as was also reported here.

Addition of Glu to primary human synovial cultures derived from RA and acute gout patients results in significantly increased levels of SF TNFa in the culture supernatants [12]. In the present study, the increase in SF Glu levels preceded SF TNFa elevations in serial collections from the patient with acute pseudogout (Figure 1). Activation of Glu receptors on human cultured clonal human synoviocytes resulted in significantly increased culture supernatant levels of RANTES (regulated on activation, normal $\mathrm{T}$ cell expressed and secreted [13], IL-8, [14] and IL-6 [15]. Studies with experimental arthritis models have demonstrated improved joint inflammatory parameters and nociceptive behaviours with Glu receptor antagonist treatments applied at several specific central and peripheral sites [2,3,16,17]. Taken together, these studies support direct and indirect consequences of SF EAA in the human arthritic process that may be independent of some inflammatory mediators.

The major limitations of this study are the small number of subjects and SF samples that were available with clinical presentations. Also, there were clinical descriptions of the active arthritis, but no formal measurements other than mild to severe pain levels reported at physical exam and mediator levels derived from the SF samples. Additional studies in human and experimental models will be needed to confirm temporal fluctuations of SF EAA and other mediator levels and their relevance in human arthritis and clinical disease activity. The patient with acute pseudogout presented with two distinct clinical courses and 
of interest, different responses to treatment and resolution. Current animal models have provided evidence of fluctuating SF mediators, over short time courses [16-18]. It might be desirable to study and compare SF EAA levels and receptor activation in distinct arthritic processes from two unrelated experimental arthropathy models at the same time, e.g. septic joint and meniscal tear in the context of joint measurements, behavioural parameters, inflammatory cytokines and localized responses to treatment.

\section{Conclusion}

This study presented very unique human arthritis courses and provided additional support that the joint microenvironment and inflammatory profile can be independent of other joints in the same patient. This also added to a body of literature that addresses glutamate and other mediators in supportive soft tissues of the joint [19]. A formal correlation of temporal fluctuations of SF mediators and clinical parameters in arthritis would provide insight into development of novel therapeutic regimens that could more effectively treat pain and inflammation and possibly blunt arthritis progression. This might be especially relevant in the acute setting.

\section{Acknowledgments}

The authors wish to thank Dr. David McAdoo, Director of the Protein Chemistry Laboratory for laboratory support and Pat Gazzoli for assistance in preparation of the figures.

\section{Grant support}

This work was supported by NIH NS 32778: Central Control of Pain and Arthritis (KNW, TAM), NIH NS 32118, Molecular Basis of Glutamate Receptor Activation, (KNW, TAM), Charles A. Dana Foundation, Clinical Hypotheses in Neuroimmunology, (TAM, KNW), and start-up dollars at the University of New Mexico.

\section{References}

1. Sluka KA, Westlund-High KN (2009) Neurologic Regulation of Inflammation. In: Kelley's Textbook of Rheumatology (8th Edn) Firestein GS, Budd RC, Harris Jr ED, McInnes IB, Ruddy S (Eds) Saunders Elsevier, Philadelphia, PA.

2. Lawand NB, Willis WD, Westlund KN (1997) Excitatory amino acid receptor involvement in peripheral nociceptive transmission in rats. Eur J Pharmacol 324: 169177.

3. Lawand NB, McNearney T, Westlund KN (2000) Amino acid release into the knee joint: key role in nociception and inflammation. Pain 86: 69-74. [Crossref]
4. McNearney T, Speegle D, Lawand N, Lisse J, Westlund KN (2000) Excitatory amino acid profiles of synovial fluid from patients with arthritis. J Rheumatol 27: 739-745. [Crossref]

5. Lawand NB, Reddig WJ, Cashin AE, Westlund KN, Willis WD (2004) NMDA receptors and associated signaling pathways: a role in knee joint blood flow regulation. Eur J Pharmacol 499: 155-161. [Crossref]

6. Carlton SM, McNearney T, Cairns BE (2003) The role of glutamate receptors in the periphery. Proceedings of the 10th World Conference on Pain 24: 125-139.

7. Primer on the Rheumatic Diseases (2001) In: John H Klippel (Ed) Arthritis Foundation, Atlanta, GA p. 353.

8. Zhang W, Doherty M, Bardin T, Barskova V, Guerne PA, et al. (2011) European league against rheumatism recommendations for calcium pyrophosphate deposition. Part I: terminology and diagnosis. Ann Rheum Dis 70: 563-570.

9. Zhang W, Doherty M, Pascual E, Barskova V, Guerne PA, et al. (2011) EULAR recommendations for calcium pyrophosphate deposition. Part II: management. Ann Rheum Dis 70: 571-575.

10. Brennan KE, McGrory BJ (2011) Monoarticular pseudogout of the hip: a case report Hosp Pract (1995) 39: 74-77. [Crossref]

11. McNearney TA, Westlund KN (2013) Excitatory amino acids display compartmental disparity between plasma and synovial fluid in clinical arthropathies. Int J Clin Exp Pathol 6: 492-497.

12. McNearney T, Baethge BA, Cao S, Alam R, Lisse JR, et al. (2004) Excitatory amino acids, TNF-alpha, and chemokine levels in synovial fluids of patients with active arthropathies. Clin Exp Immunol 137: 621-627.

13. McNearney TA, Ma Y, Chen Y, Taglialatela G, Yin H, et al. (2010) A peripheral neuroimmune link: glutamate agonists upregulate NMDA NR1 receptor mRNA and protein, vimentin, TNF-alpha, and RANTES in cultured human synoviocytes. $\mathrm{Am} J$ Physiol Regul Integr Comp Physiol 298: R584-598. [Crossref]

14. Liu L, Xue Y, Zhu Y, Xuan D, Yang X, et al. (2016) Interleukin 37 limits monosodium urate crystal-induced innate immune responses in human and murine models of gout. Arthritis Res Ther 18: 268.

15. Flood S, Parri R, Williams A, Duance V, Mason D (2007) Modulation of interleukin-6 and matrix metalloproteinase 2 expression in human fibroblast-like synoviocytes by functional ionotropic glutamate receptors. Arthritis Rheum 56: 2523-2534.

16. Ren K, Williams GM, Hylden JLK, Ruda MA, Dubner R (1992) The intrathecal administration of excitatory amino-acid receptor antagonists selectively attenuated carrageenan-induced behavioral hyperalgesia. Eur J Pharmacol 219: 235-243.

17. Sluka KA, Westlund KN (1993) Centrally administered non-NMDA but not NMDA receptor antagonists block peripheral knee joint inflammation. Pain 55: 217-225.

18. Szekanecz Z, Halloran MM, Volin MV, Woods JM, Strieter RM, et al. (2000) Temporal expression of inflammatory cytokines and chemokines in rat adjuvant-induced arthritis. Arthritis Rheum 43: 1266-1277.

19. Alfredson H, Lorentzon R (2003) Intratendinous glutamate levels and eccentric training in chronic Achilles tendinosis: a prospective study using microdialysis technique. Knee Surg Sports Traumatol Arthrosc 11: 196-199.

Copyright: (C2018 McNearney TA. This is an open-access article distributed under the terms of the Creative Commons Attribution License, which permits unrestricted use, distribution, and reproduction in any medium, provided the original author and source are credited. 